\title{
Clodronate Disodium
}

National Cancer Institute

\section{Source}

National Cancer Institute. Clodronate Disodium. NCI Thesaurus. Code C1418.

The disodium salt of a nitrogen-free bisphosphonate analog of naturally occurring pyrophosphate. Clodronate binds to calcium and inhibits osteoclastic bone resorption and hydroxyapatite crystal formation and dissolution, resulting in a reduction of bone turnover. This agent may control malignancy-associated hypercalcemia, inhibit osteolytic bone metastasis and decrease pain. 